\section{Zellzahlen, quantitative}

H. Baum

Institut für Laboratoriumsmedizin, Mikrobiologie und

Blutdepot, Regionale Kliniken Holding RKH GmbH,

Ludwigsburg, Deutschland

Englischer Begriff cell count, quantitative

Definition Absolutzellzahl der Leukozytensubpopulationen bei der morphologischen Differenzierung.

Beschreibung Die morphologische Differenzierung eines Blutausstriches ergibt primär nur die relative Verteilung der einzelnen Subpopulationen wieder. Entscheidend für die Diagnostik ist jedoch in der Regel die absolute Zellzahl der einzelnen Subpopulationen pro Volumeneinheit, da die relative Verteilung stark von der Gesamtleukozytenzahl abhängig ist. So darf die Angabe einer Verminderung oder Vermehrung einer Subpopulation nur in Bezug auf die absolute Zellzahl der einzelnen Subpopulationen erfolgen. In der Tabelle sind die Referenzwerte ( $\triangleright$ Referenzwert) für die absoluten Zellzahlen der Leukozytensubpopulationen im peripheren Blut für verschiedene Altersstufen zusammengefasst (nach: Koeppen und Heller 1991):

\begin{tabular}{|l|l|l|}
\hline Alter & Zellart & $\begin{array}{l}\text { Absolute Zellzahl (G/L) } \\
\text { Median (Streubreite) }\end{array}$ \\
\hline Bei & Leukozyten & $\mathbf{1 8 , 0}(\mathbf{9 , 0}-\mathbf{3 0 , 0})$ \\
Geburt & Neutrophile Granulozyten & $11,0(6,0-26)$ \\
& • stabförmige & 1,6 \\
& • segmentförmige & 9,4
\end{tabular}

(Fortsetzung)

\begin{tabular}{|c|c|c|}
\hline Alter & Zellart & $\begin{array}{l}\text { Absolute Zellzahl (G/L) } \\
\text { Median (Streubreite) }\end{array}$ \\
\hline & $\begin{array}{l}\text { Eosinophile Granulozyten } \\
\text { Basophile Granulozyten } \\
\text { Lymphozyten } \\
\text { Monozyten }\end{array}$ & $\begin{array}{l}0,4(0,02-0,85) \\
0,1(0-0,64) \\
5,5(2,0-11,0) \\
1,5(0,4-3,1)\end{array}$ \\
\hline $1 \mathrm{Jahr}$ & $\begin{array}{l}\text { Leukozyten } \\
\text { Neutrophile Granulozyten } \\
\text { - stabförmige } \\
\text { - segmentförmige } \\
\text { Eosinophile Granulozyten } \\
\text { Basophile Granulozyten } \\
\text { Lymphozyten } \\
\text { Monozyten }\end{array}$ & $\begin{array}{l}\mathbf{1 1 , 4}(\mathbf{6 , 0}-\mathbf{1 7 , 0 )} \\
3,5(1,5-8,5) \\
0,35 \\
3,2 \\
0,3(0,05-0,7) \\
0,05(0-0,2) \\
7,0(4,0-10,5) \\
0,55(0,05-1,1)\end{array}$ \\
\hline $\begin{array}{l}10 \\
\text { Jahre }\end{array}$ & $\begin{array}{l}\text { Leukozyten } \\
\text { Neutrophile Granulozyten } \\
\text { - stabförmige } \\
\text { - segmentförmige } \\
\text { Eosinophile Granulozyten } \\
\text { Basophile Granulozyten } \\
\text { Lymphozyten } \\
\text { Monozyten }\end{array}$ & $\begin{array}{l}\mathbf{8 , 1}(\mathbf{4 , 5}-\mathbf{1 3 , 5 )} \\
4,4(1,8-8,0) \\
0,24(0-1,0) \\
4,2(1,8-7,0) \\
0,2(0-0,6) \\
0,04(0-0,2) \\
3,1(1,5-6,5) \\
0,35(0-0,8)\end{array}$ \\
\hline $\begin{array}{l}21 \\
\text { Jahre }\end{array}$ & $\begin{array}{l}\text { Leukozyten } \\
\text { Neutrophile Granulozyten } \\
\text { • stabförmige } \\
\text { - segmentförmige } \\
\text { Eosinophile Granulozyten } \\
\text { Basophile Granulozyten } \\
\text { Lymphozyten } \\
\text { Monozyten }\end{array}$ & $\begin{array}{l}\mathbf{7 , 4}(\mathbf{4 , 5}-\mathbf{1 1 , 0 )} \\
4,4(1,8-7,7) \\
0,22(0-0,7) \\
4,2(1,8-7,0) \\
0,2(0-0,45) \\
0,04(0-0,2) \\
2,5(1,0-4,8) \\
0,3(0-0,8)\end{array}$ \\
\hline
\end{tabular}

\section{Literatur}

Koeppen KM, Heller S (1991) Differentialblutbild (panoptische Färbung. In: Boll I, Heller S (Hrsg) Praktische Blutzelldiagnostik. Springer, Berlin, S 178-179 